\title{
Content-Based Retrieval and Classification of Ultrasound Medical Images of Ovarian Cysts
}

\author{
Abu Sayeed Md. Sohail ${ }^{1}$, Prabir Bhattacharya ${ }^{2}$, Sudhir P. Mudur ${ }^{1}$, \\ Srinivasan Krishnamurthy ${ }^{3}$, and Lucy Gilbert ${ }^{3}$ \\ ${ }^{1}$ Dept. of Computer Science and Software Engineering, Concordia University, Canada \\ \{a_sohai, mudur\} @encs. concordia.ca \\ ${ }^{2}$ Dept. of Computer Science, University of Cincinnati, Ohio, USA \\ bhattapr@ucmail.uc.edu \\ ${ }^{3}$ Dept. of Obstetrics and Gynecology, Royal Victoria Hospital, Montreal, Canada \\ \{srinivasan.krishnamurthy, lucy.gilbert\}@muhc.mcgill.ca
}

\begin{abstract}
This paper presents a combined method of content-based retrieval and classification of ultrasound medical images representing three types of ovarian cysts: Simple Cyst, Endometrioma, and Teratoma. Combination of histogram moments and Gray Level Co-Occurrence Matrix (GLCM) based statistical texture descriptors has been proposed as the features for retrieving and classifying ultrasound images. To retrieve images, relevance between the query image and the target images has been measured using a similarity model based on Gower's similarity coefficient. Image classification has been performed applying Fuzzy $k$-Nearest Neighbour $(k-\mathrm{NN})$ classification technique. A database of 478 ultrasound ovarian images has been used to verify the retrieval and classification accuracy of the proposed system. In retrieving ultrasound images, the proposed method has demonstrated above $79 \%$ and $75 \%$ of average precision considering the first 20 and 40 retrieved images respectively. Further, 88.12\% of average classification accuracy has been achieved in classifying ultrasound images using the proposed method.
\end{abstract}

Keywords: Ultrasound Medical Image Retrieval, Ovarian Cyst Classification, Texture Feature, Histogram Moments, Fuzzy $k$-NN.

\section{Introduction}

With advancements in image processing and pattern classification techniques, ContentBased Image Retrieval (CBIR) has become one of the most active research topics of computer vision during the last 15-years. As medical images are produced in large number everyday during regular clinical practice, they have been frequently used in developing and analyzing the performance of such retrieval systems. This has eventually lead medical domain to be cited as one of the principal application domains of CBIR technologies in terms of potential for high impact. A large number of propositions have already been made for content-based retrieval of medical images including radiology images, X-ray images, CT images of lung, dermatology images, MRI images 
of heart and brain, ultrasound images of kidney and breast [1, 2]. However, to the best of our knowledge, no published research has yet reported the application of CBIR and image classification techniques over ultrasound images of ovarian abnormalities.

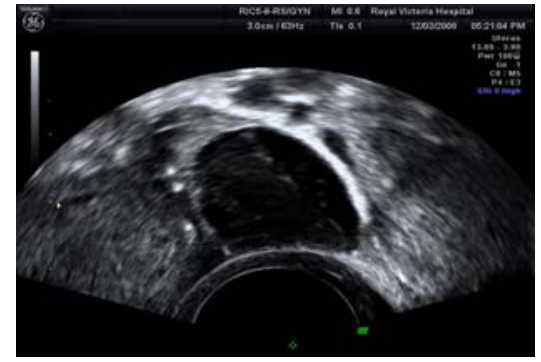

(a) Simple Cyst

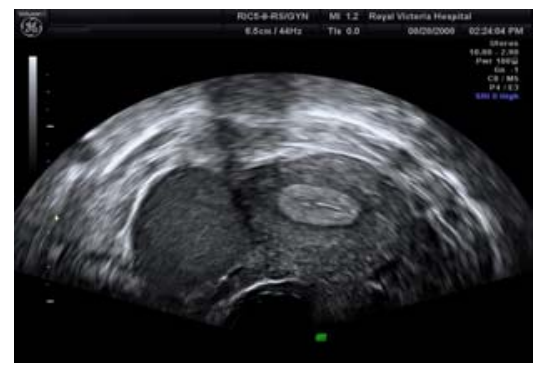

(c) Endometrioma

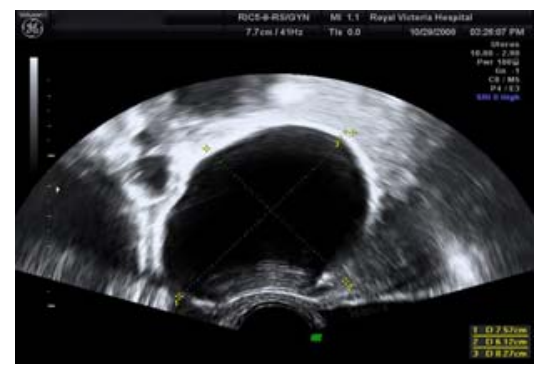

(e) Teratoma

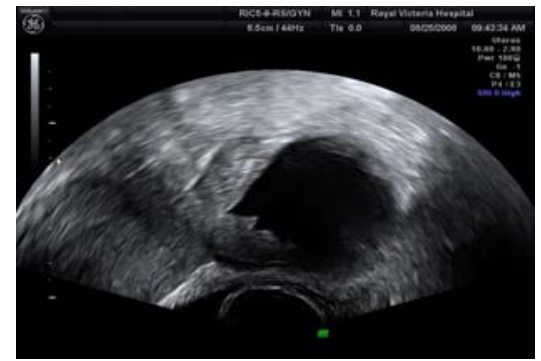

(b) Simple Cyst

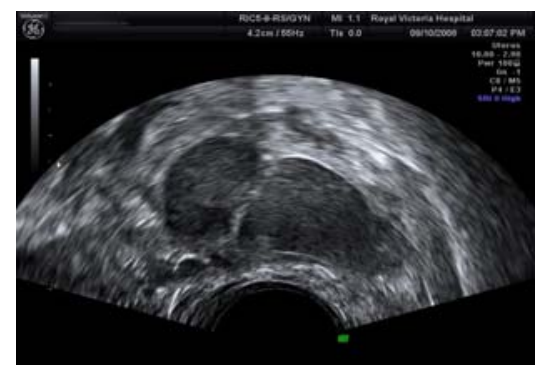

(d) Endometrioma

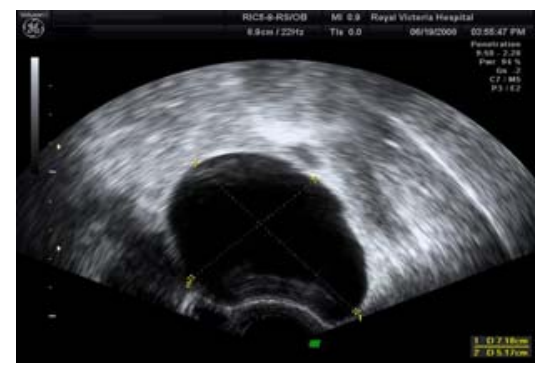

(f) Teratoma

Fig. 1. Sample ultrasound images representing three types of ovarian cysts: Simple Cyst, Endometrioma, and Teratoma

Ovarian cysts are particularly heterogeneous in nature (Fig. 1) and profiling them accurately is very important in order to arrive at a diagnosis. During the past decade, visual ultrasound examination has been accepted as the optimal diagnostic modality for the non-invasive assessment of ovarian cysts and other types of ovarian abnormalities [3]. Although, several ultrasound-based algorithms have been proposed for this purpose, recognition of inherent patterns through visual observation of ultrasound images remains the best way for assessing their nature and category. However, this method largely depends on the accumulation of practical experience in identifying the 
morphology and characteristics of various types of ovarian cysts present in their corresponding ultrasound images. Therefore, inexperienced ultrasonographers and gynecologists always face difficulties in differentiating among different types of cysts resulting in lower rate of correct diagnosis. Since incorrect diagnosis can either result in unnecessary biopsies/surgery, or worse, missed cases, there is a huge need for inexperienced operators to be given supporting tools to help increase their diagnostic accuracy. The proposed system for retrieval and classification of ultrasound images could serve the purpose of such a decision support tool in the diagnosis of ovarian abnormalities.

The subsequent discussions of the paper have been organized as follows: methods for calculating and combining histogram moments and GLCM based texture feature have been explained in Section 2. Section 3 provides a brief theoretical overview covering the similarity matching and image classification techniques applied. Section 4 demonstrates the experimental results achieved applying the proposed method in retrieving and classifying ultrasound images of ovarian cysts, and finally, Section 5 concludes the paper.

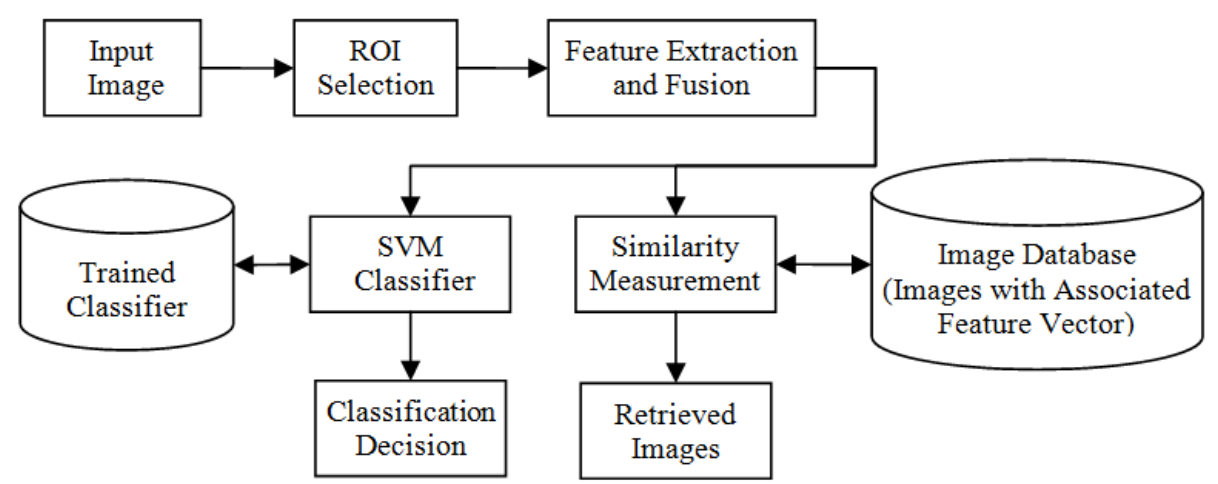

Fig. 2. Flowchart of the proposed system for retrieval and classification of ultrasound ovarian images

\section{Feature Extraction}

The proposed method of ultrasound ovarian image retrieval and classification works in three main stages: (i) feature extraction and fusion, (ii) image retrieval by similarity measurement, and (iii) image classification using Fuzzy $k$-NN. Since automated segmentation of ultrasound images is still an open problem, the proposed method requires manual intervention in selecting the Region of Interest (ROI) for feature extraction from an ultrasound image. Once the region has been specified using a number of points on the image surface, the rest of the process of feature extraction, feature fusion, image retrieval and classification is done automatically without further intervention from the user. The flowchart of the proposed system is given in Fig. 2. 
In this work, the following 2-types of features have been extracted from ultrasound images for the purpose of their retrieval and classification:

(a) Histogram Moments

(b) GLCM Based Statistical Texture Descriptors

\subsection{Histogram Moments}

Histogram based feature is one of the most widely used visual features in image retrieval and classification. The histogram of an image $f_{n}$ is an $N$-dimensional vector $H\left(f_{n}, i\right) ; i=0,1,2, \cdots, N-1$, where $N$ is the number of gray levels and $H\left(f_{n}, i\right)$ is the number of pixels with gray level value $i$. Histogram feature is relatively robust to background complication. Besides, it is also insensitive to changes such as image size, rotation and slight transition, each of which has little or no impact on the distribution of the gray levels in an image. However, the disadvantage of using histogram feature is that different images can have similar overall histogram in a large image database. Consequently, this can lead to poor performance in terms of retrieval and classification accuracy. Another drawback of using histogram features for ultrasound image classification is that an ultrasound image can have histogram with many empty bins due to the quantization process involved in the imaging system. As a result, slight changes in illumination may cause a shift in the histogram $p d f$, which can introduce huge changes between the set of features obtained from two similar images. The moment-based approach can minimize these problems since it smoothes the gram $p d f$. The histogram distribution of an image can be interpreted as a probability distribution and can be characterized by its moments. Stricker and Orengo [4] introduced a method using the central moment approach. Mandal et al. [5] used orthogonal Legendre moments for histogram indexing, which resulted in a better performance than regular or central moments. Legendre moments are based on orthogonal Legendre polynomials. A Legendre polynomial is defined as follows:

$$
P_{k}(x)=\frac{1}{k ! 2^{k}} \frac{d^{k}}{d x^{k}}\left(x^{2}-1\right)^{k}=\sum_{j=0}^{k} a_{k j} x^{j}
$$

The value of $a_{k j}$ can be expressed as:

$$
a_{k j}=\sum_{\substack{j=0 \\ j, k \text { odd } \\ j, k \text { even }}}^{k}(-1)^{(k-j) / 2} \frac{(k+j) !}{2^{k}\left(\frac{k-j}{2}\right) !\left(\frac{k+j}{2}\right) ! j !}
$$

The $k$-th Legendre moment of a function $f(x)$ is defined by

$$
\lambda_{k}=\frac{2 k+1}{2} \int_{-\infty}^{\infty} P_{k}(x) f(x) d x \quad k \geq 0, k \in \mathbb{Z}
$$

Replacing the value of $P_{k}(x)$ from Eq. (1), and applying the definition of centrals moments, $\lambda_{k}$ can be expressed as: 


$$
\lambda_{k}=\frac{2 k+1}{2} \sum_{j=0}^{k} a_{k j} M_{j}
$$

It can be observed that the Legendre moment of any order depends only on the regular moments of the same order and lower and can be calculated easily using the above equation.

Generally, the image grey levels range from 0 to 255. Since, the Legendre polynomials are orthogonal only in the interval $[-1,1]$, the dynamic range of the $p d f$ has to be mapped onto this interval. The histogram $p d f$ function $f(x)$ can then be written as an infinite series expansion in terms of the Legendre polynomials as:

$$
f(x)=\sum_{k=0}^{\infty} \lambda_{k} P_{k}(x)
$$

From this equation, the image $p d f$ can be reconstructed using first $(N+1)$ moments as follows:

$$
f^{\prime}(x)=\sum_{k=0}^{N} \lambda_{k} P_{k}(x)
$$

The reconstructed $p d f$ function $f^{\prime}(x)$ is then free from any quantization effect and has no empty bins. The optimum number of moments required for reconstructing the image $p d f$ accurately is an important concern. Usually, 10-16 moments can give a good representation of an image $p d f$ when it does not contain any sharp peak [5]. Ultrasound ovarian images usually contain sharp peaks with empty bins and therefore cannot be represented well using a small number of moments. To quantify the reconstruction efficiency as well as the no. of moments required to better represent ultrasound images, we have calculated the Signal to Error Ratio (SER) as follows [5]:

$$
S E R=\frac{\int f^{2}(x) d x}{\int\left[f(x)-f^{\prime}(x)\right]^{2} d x}
$$

Where, $f(x)$ and $f^{\prime}(x)$ are the original histogram and the moment reconstructed histogram respectively. Table 1 shows the percentage of images having a $S E R$ greater than a certain threshold value using 8, 16, 32, 64, 128 moments.

Table 1. $S E R$ of the re-constructed image histogram $p d f$ with finite number of moments. Using 128 moments, $98 \%$ of the reconstructed histograms have $S E R>8 d B$.

\begin{tabular}{|c|c|c|c|c|c|}
\hline & \multicolumn{5}{|c|}{ No. of Moments } \\
\hline$S E R$ & 8 & 16 & 32 & 64 & 128 \\
\hline$>8 \mathrm{~dB}$ & $52 \%$ & $79 \%$ & $91 \%$ & $97 \%$ & $98 \%$ \\
\hline$>9 \mathrm{~dB}$ & $41 \%$ & $72 \%$ & $86 \%$ & $97 \%$ & $97 \%$ \\
\hline$>10 \mathrm{~dB}$ & $35 \%$ & $67 \%$ & $80 \%$ & $93 \%$ & $95 \%$ \\
\hline$>11 \mathrm{~dB}$ & $31 \%$ & $63 \%$ & $77 \%$ & $91 \%$ & $94 \%$ \\
\hline$>12 \mathrm{~dB}$ & $25 \%$ & $57 \%$ & $72 \%$ & $88 \%$ & $92 \%$ \\
\hline
\end{tabular}


As can be observed from Table 1, use of 64 and 128 moments demonstrates the best re-construction capability. However, calculation of 128 moments is computationally at least twice as expensive as calculating 64 moments. In addition, use of 64 moments instead of 128 has a significant impact on reducing the dimension of the extracted feature vector. Therefore, we opted for using the first 64 moments as image histogram based features.

\subsection{GLCM Based Texture Feature}

The co-occurrence probabilities of GLCM provide a second-order method for generating texture features [6]. These probabilities represent the conditional joint probabilities of all pair wise combinations of grey levels in the spatial window of interest with respect to two parameters: inter-pixel distance $(d)$ and orientation $(\theta)$. The probability measure can be defined as: $P(x)=\left\{C_{i j} \mid(d, \theta)\right\}$, where $C_{i j}$, the co-occurrence probability between grey level $i$ and $j$, is defined as:

$$
C_{i j}=\frac{P_{i, j}}{\sum_{i, j=1}^{G} P_{i, j}}
$$

Here $P_{i, j}$ represents the number of occurrences of grey level $i$ and $j$ within a specific window, given a certain pair of $(d, \theta)$; and $G$ is the quantized number of grey levels. The sum in the denominator thus represents the total number of grey level pairs $(i, j)$ within the window. For extracting GLCM based texture features from ultrasound ovarian images, we obtained four co-occurrence matrices from each image using $\theta=\{0,45,90,135\}$ degree and $d=1$ pixel. After that, 19-statistical texture descriptors have been calculated from each of these co-occurrence matrices as proposed in [6] and [7]. These descriptors are: angular second moment (energy), contrast, correlation, sum of squares, inverse difference moment, sum average, sum variance, sum entropy, entropy, difference variance, difference entropy, two information measures of correlation, maximal correlation coefficient, autocorrelation, dissimilarity, cluster shade, cluster performance, and maximum probability. Then, by taking the range statistics (maximum and minimum), and average of these texture descriptors calculated using the four GLCMs, a total of $19 \times 3=57$ texture features were extracted from each of the images and used for their retrieval and classification.

\subsection{Feature Fusion and Normalization}

After extracting the histogram moments and GLCM based texture features from an image, they are organized into a single feature vector. Each feature vector $x_{k}$, consisting of 121 features $(64+57)$, is then normalized as:

$$
\hat{x}_{k}=\frac{x_{k}-\mu_{k}}{\sigma_{k}}
$$

where, $\mu_{k}$ and $\sigma_{k}$ are the mean and standard deviation of feature vector $x_{k}$. 


\section{Image Retrieval and Classification}

\subsection{Similarity Model for Image Retrieval}

For retrieving ultrasound images, Gower's similarity coefficient [8] based similarity model has been used in which combination of features to constitute a global similarity is done as an average of each of the individual similarities on each feature. The model is defined as follows [9]:

$$
G S_{i j}=\frac{\sum_{k=1}^{n} S_{i j}^{(k)}}{\sum_{k=1}^{n} \delta_{i j}^{(k)}}
$$

Here, $S_{i j}^{(k)}$ is the result of comparing image $i$ and $j$ on their feature $k$, and $\delta_{i j}^{(k)}$ represents the possibility of comparing image $i$ and $j$ on their feature $k$. In Eq. (2), $\delta_{i j}^{(k)}=1$ if image $i$ and $j$ can be compared on feature $k$, otherwise, $\delta_{i j}^{(k)}=0$. If the image $i$ and image $j$ can be compared across all the considered features, $\sum_{k=1}^{n} \delta_{i j}^{(k)}=$ $N$, which is the dimension of the feature vector. So, global similarity $G S_{i j}$ between images $i$ and $j$ is defined as an average of the similarities on each feature between image and $i$ and $j$. The quantity $S_{i j}^{(k)}$ can be defined as follows:

$$
S_{i j}^{(k)}=1-\frac{\left|x_{i k}-x_{j k}\right|}{R_{k}}
$$

Where $R_{k}$ represents a normalization factor and is calculated as: $R_{k}=\operatorname{Max}\left(x_{i k}\right)-$ $\operatorname{Min}\left(x_{i k}\right)$ where $x_{i k} ; i=1,2, \cdots, n$ is the set of values taken by each of the image $i$ of the sample considered for the feature $k . S_{i j}^{(k)}=1$ if image $i$ and $j$ are identical and $S_{i j}^{(k)}=0$ if they are completely different. $S_{i j}^{(k)}$ can take a positive value between 0 and 1 if the two images have a certain degree of similarity according to feature $k$. Using Eq. (3) and considering that all features can be compared, global similarity $G S_{i j}$ between two images $i$ and $j$, as defined in Eq. (2), can be re-written as:

$$
G S_{i j}=\frac{1}{N} \sum_{k=1}^{n}\left(1-\frac{\left|x_{i k}-x_{j k}\right|}{R_{k}}\right)
$$

\subsection{Image Classification Using Fuzzy $k$-NN}

Let $X \subseteq \mathfrak{R}^{N}$ be the set of all possible input patterns, and $\chi=\left\{x_{1}, x_{2}, \cdots, x_{n}\right\} \subseteq X$ be a set of input training patterns for which the corresponding class labels are already known. In the conventional $k-\mathrm{NN}$ algorithm, the Euclidean distances between the test pattern and all training patterns are calculated, and the test pattern is assigned the class label that most of the $k$-closest training patterns have [10]. Let the Euclidean distance between the test pattern $y \in X \subseteq \Re^{N}$ and the training pattern $x_{j} \in \mathcal{X}$ be denoted by $\left\|y-x_{j}\right\|=\sqrt{\sum_{i=1}^{N}\left(y_{i}-x_{j i}\right)^{2}}$. Since the number of training patterns is $n$, a 
total of $n$ such distances are calculated, and the closest $k$-training patterns are identified as neighbors. The output of the conventional $k$-NN algorithm attains a richer semantic when the output is interpreted as a posteriori probability [11]. Hence, instead of labeling the output class label equal to the class label that most of the neighbors have, the following class confidence values are assigned to the test pattern $y$ :

$o_{c}(y)=\frac{1}{k}$ (no. of neighbors with class label $\left.c\right) ; \forall_{c}$

$=\frac{1}{k} \sum_{j=1}^{k} \delta_{c}\left(x_{j}\right) ; \forall_{c}$

where $\delta_{c}\left(x_{j}\right)$ is the characteristic function corresponding to the $j$-th neighbor, i.e., $\delta_{c}\left(x_{j}\right)=1$ if $x_{j}$ has the class label $c$, and $\delta_{c}\left(x_{j}\right)=0$ otherwise. Here, $o_{c}(y)$ is the posteriori probability that $y$ belongs to the class $c$. With this formulation, we can still derive the hard decision by assigning the class label $j$ to the test pattern $y$ where $o_{j}(y)=\max _{1,2, \cdots, \mathcal{C}}\left\{o_{c}(y)\right\}$ and $\mathcal{C}$ is the total number of classes.

In the conventional $k$-NN algorithm, all $k$ neighbors receive equal importance, and the class label of each training pattern is considered crisp. The fuzzy $k$-NN algorithm refines the conventional $k$-NN algorithm-

1. By weighing the contribution of each of the $k$ neighbors based on its distance to the test pattern. Evidently, the closest neighbor should receive the highest weight. Hence, the $i$-th closest neighbor is weighted based on its relative distance with respect to all $k$-closest neighbors. Thus, the relative weight of the $i$-th neighbor $i \in\{1,2, \ldots, k\}$ is $\frac{1 /\left\|y-x_{i}\right\|^{2 /(q-1)}}{\sum_{j=1}^{k}\left(1 /\left\|y-x_{j}\right\|^{2 /(q-1)}\right)}$; where $\left\|y-x_{i}\right\|$ is the Euclidean distance between $y$ and $x_{i}$; and $q$ determines how strongly the distance is weighted while calculating each neighbor's contribution to the membership value. Here, the denominator is used for normalization such that the sum of the weights $=1$.

2. By considering that each neighbor may belong to more than one class. It typically happens where the classes overlap. Hence, the crisp class membership of each training pattern, i.e., $\delta$, is modified to the fuzzy membership function $\mu$.

To consider the above two refinements, Eq. (5) is modified to the following [12]:

$$
o_{c}(y)=\sum_{i=1}^{k}\left(\frac{\frac{1}{\left\|y-x_{i}\right\|^{2 /(q-1)}}}{\sum_{j=1}^{k} \frac{1}{\left\|y-x_{j}\right\|^{2 /(q-1)}}}\right) \mu_{\mathcal{C}_{c}}\left(x_{i}\right)
$$

Where $\mu_{\mathcal{C}_{c}}\left(x_{i}\right)$ is the fuzzy membership of the $i$-th neighbor in the $c$-th class. In this case $o_{c}(y)$ is interpreted as the fuzzy membership function [13]. 
In the fuzzy $k$-NN algorithm, the initial membership on each training pattern can be assigned in the following two ways $[12,14]$ :

1. Crisp Membership: Each training pattern can have complete membership in their known class and non-memberships in all other classes.

2. Constrained Fuzzy Membership: The $k$-nearest neighbors of each training pattern (say $x_{i}$ ) are found, and the membership of $x_{i}$ in each class is assigned as:

$$
\mu_{\mathcal{C}_{c}}\left(x_{i}\right)= \begin{cases}0.51+\frac{0.49 n_{j}}{k} & \text { if } j=c, \\ \frac{0.49 n_{j}}{k} & \text { If } j \neq c\end{cases}
$$

The value $n_{j}$ is the number of the neighbors found that belongs to the $j$-th class. This initialization technique fuzzifies the memberships of the labeled samples that are in the region where classes are overlapping. Moreover, the patterns that are well away from the overlapping area are assigned with the complete membership in the known class. Consequently, a test pattern lying in the overlapping region will be influenced to a lesser extent by the labelled samples that are also in the overlapping area [14].

\section{Performance Analysis}

Performance of the proposed method of ultrasound ovarian image retrieval and classification has been tested using 478 ultrasound images of ovarian cysts collected during regular clinical practice at the Department of Obstetrics and Gynecology, Royal Victoria Hospital, Montreal. The collected images were classified into three types of ovarian cyst: Simple Cyst (187 images), Endometrioma (154 images) and Teratoma (137 images). This categorization was performed by at least one expert and the categorization decision was verified by consulting the proven pathological diagnosis associated with the respective ultrasound ovarian images.

\subsection{Retrieval Performance}

To evaluate the retrieval performance, randomly selected 50 images of each category have been used as the query images. We adopted "Query by Example" for submitting the query to the retrieval system where the query is specified by providing an example image to the system. A retrieved image is considered a match if it belongs to the same category as that of the query image. For quantitative evaluation, retrieval performances of each category (simple cyst, endometrioma, and teratoma) were compared by calculating "Precision" values for $N=\{10,20,30,40,50,60,70,80,90,100$, $120,140\}$ retrieved results as:

$$
\text { Precision }=\frac{\text { True Positive }}{\text { True Positive }+ \text { False Positive }}
$$

Fig 3. demonstrates the precision curves drawn by calculating the average precision values from the retrieved images of each category. As can be observed from this graph, the best overall retrieval performance has been achieved in retrieving the ultrasound 
images of simple cyst. The average precision value lies above $79 \%$ for the first 20 retrieved images and above $75 \%$ for the first 40 retrieved images, which indicates very satisfactory and consistent retrieval performance.

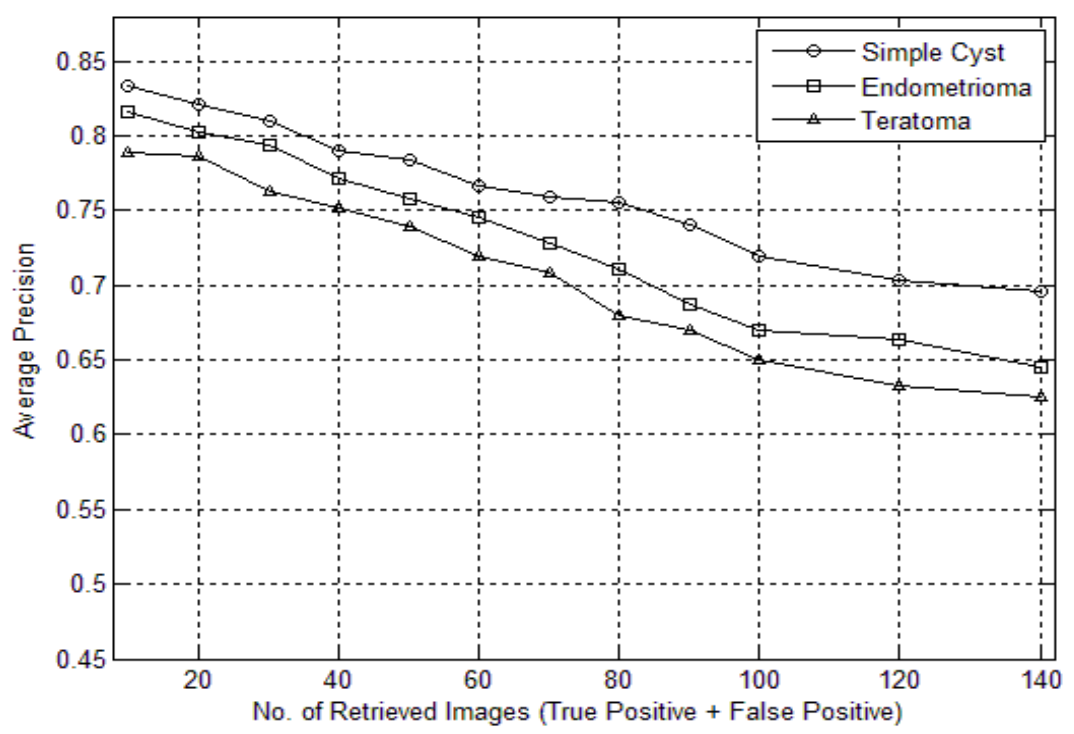

Fig. 3. Performance of the proposed method in retrieving ultrasound images of ovarian cysts

\subsection{Classification Performance}

Due to its capability of providing high classification accuracy over small training sets as well as comparatively better generalization performance on data that belongs to a limited number of classes, fuzzy $k$-Nearest Neighbor (fuzzy $k$-NN) classification technique has been chosen in this work for classifying ultrasound images into 3categories: simple cyst, endometrioma, and teratoma. The initial membership on each training pattern was assigned using Constrained Fuzzy Membership as defined by Eq. (6). The choice of the number of nearest neighbors $(k)$ is the most important customizations that can be made while adjusting fuzzy $k$-NN classifier to a particular application domain. By performing experiments with fuzzy $k$-NN applying different values of $k$ starting with $k=3$, we found that a stable average classification accuracy of $88.12 \%$ was achieved using $k=21$ (Fig . 4). Feature extracted from 200 images of the databases has been used to train the classifier applying " $K$-Fold Cross Validation" technique with $K=5$. Performance of the proposed ultrasound image classification method has also been compared with other popular classification techniques namely, SVM (with RBF, Sigmoid and Polynomial kernels), ordinary $k$-Nearest Neighbor ( $k$ $\mathrm{NN}$ ) and Neural Network (NN). Results regarding these comparisons have been summarized in Table 2. 


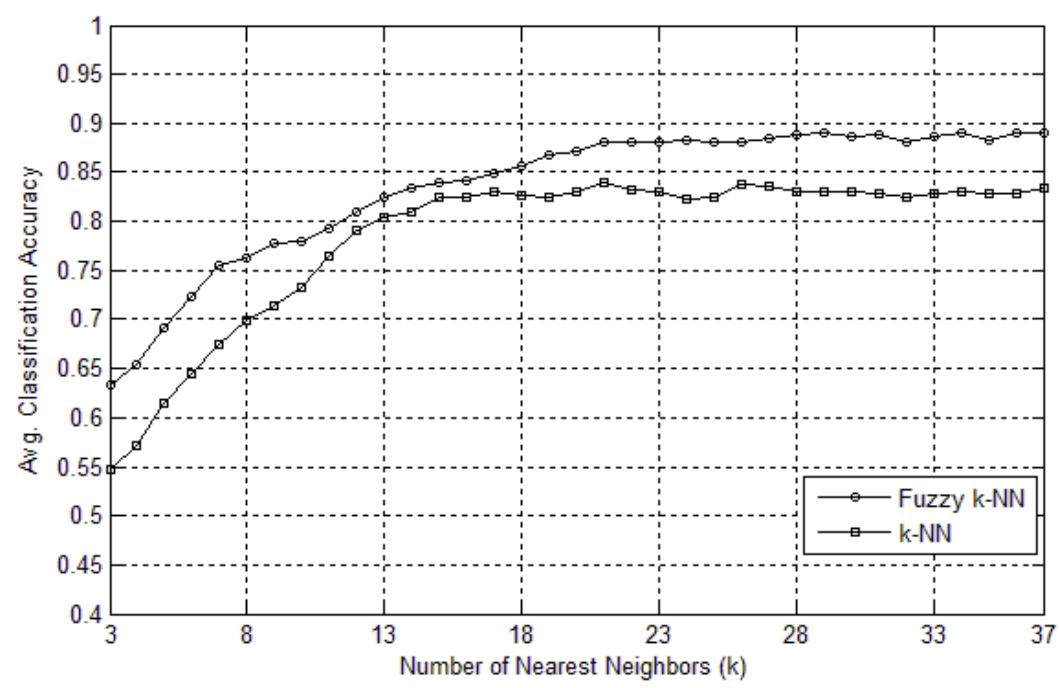

Fig. 4. Selection of a suitable value of $k$ (no. of nearest neighbors) for Fuzzy $k$-NN and ordinary $k$-NN classifier

Table 2. Comparison of classification accuracy (\%) achieved using different classifiers in classifying ultrasound ovarian images

\begin{tabular}{|l|c|c|c|c|}
\hline & Simple Cyst & Endometrioma & Teratoma & Average \\
\hline Fuzzy $k$-NN & 91.44 & 88.96 & 83.94 & 88.12 \\
\hline$k$-NN & 86.10 & 80.52 & 81.02 & 82.55 \\
\hline SVM (RBF Kernel) & 88.77 & 86.36 & 84.67 & 86.60 \\
\hline SVM (Polynomial Kernel) & 86.63 & 83.12 & 80.29 & 83.35 \\
\hline SVM (Sigmoid Kernel) & 88.24 & 85.71 & 78.10 & 84.02 \\
\hline Neural Network & 78.07 & 74.68 & 73.72 & 75.49 \\
\hline
\end{tabular}

\section{Conclusions}

We have presented a combined method for retrieval and classification of ultrasound ovarian images using combination of histogram moments and gray level co-occurrence matrix based texture descriptors. With $88.12 \%$ of average classification accuracy and above $79 \%$ and $75 \%$ precision for the first 20 and 40 retrieved images respectively, the proposed method has been able to demonstrate significant potential in support of combining histogram moments and GLCM based texture feature for retrieving and classifying ultrasound images. In future, we plan to investigate the classification and retrieval performance of the proposed method using ultrasound images of other types of ovarian cysts. Our ultimate objective is to incorporate the proposed method in developing a Computer-Aided Diagnosis (CAD) system for providing decision support in the diagnosis of ovarian abnormalities. It is expected that by querying such a CAD system with 
new images and consulting the retrieved results along with their proven pathological diagnosis, the physician would gain more confidence in his/her decision or even sometimes see the scope of considering other possibilities towards improving their overall accuracy in the diagnosis of ovarian abnormalities.

\section{References}

1. Müller, H., Michoux, N., Bandon, D., Geissbuhler, A.: A Review of Content-Based Image Retrieval Systems in Medical Applications-Clinical Benefits and Future Directions. Journal of Medical Informatics 73(1), 1-23 (2004)

2. Lehman, T.M., Güld, M.O., Thies, C., Fischer, B., Spitzer, K., Keysers, D., Ney, H., Kohnen, M., Schubert, H., Wein, B.B.: Content-Based Image Retrieval in Medical Applications. Methods of Information in Medicine 43(4), 354-361 (2004)

3. Van Nagell, J.R., Depriest, P.D., Donaldson, E.S., Gallion, H.H., Pavlik, E.J., Kryscio, R.J.: Ovarian Cancer Screening in Asymptomatic Postmenopausal Women by Transvaginal Sonography. Cancer 68(3), 458-462 (2006)

4. Stricker, M., Orengo, M.: Similarity of Color Images. In: SPIE: Storage and Retrieval for Image and Video Databases III, vol. 2420, pp. 381-392. SPIE Publications, Bellingham (1995)

5. Mandal, M.K., Aboulnsar, T., Panchanathan, S.: Image Indexing Using Moments and Wavelet. IEEE Trans. Consumer Electronics 41, 557-565 (1996)

6. Haralick, R.M., Shanmugan, K., Dinstein, I.: Textural Features for Image Classification. IEEE Trans. Systems, Man and Cybernetics. 3(6), 610-621 (1973)

7. Soh, L.-K., Tsatsoulis, C.: Texture Analysis of SAR Sea Ice Imagery Using Gray Level Co-Occurrence Matrices. IEEE Trans. Geoscience Remote Sensing 37(2), 780-795 (1999)

8. Gower, J.C.: A General Coefficient of Similarity and Some of Its Properties. Biometrics 27(4), 857-871 (1971)

9. Abbadeni, N.: Content Representation and Similarity Matching for Texture-Based Image Retrieval. In: 5th ACM SIGMM international Workshop on Multimedia information Retrieval (MIR 2003), pp. 63-70. ACM Publications, New York (2007)

10. Mitchell, T.M.: Machine Learning. McGraw-Hill, New York (1997)

11. Duda, R., Hart, P.: Pattern Classification and Scene Analysis. Wiley, New York (1973)

12. Keller, J.M., Gray, M.R., Givens, J.A.: A Fuzzy k-Nearest Neighbor Algorithm. IEEE Trans. Systems Man Cybernetics 15(4), 580-585 (1985)

13. Klir, G.S., Yuan, B.: Fuzzy Sets and Fuzzy Logic Theory and Applications. Prentice-Hall, Englewood Cliffs (1995)

14. Sarkar, M.: Fuzzy-Rough Nearest Neighbor Algorithm in Classification. Fuzzy Sets and Systems 158, 2134-2152 (2007) 